\title{
Mathematical models of hysteresis
}

\author{
Augusto Visintin \\ Dipartimento di Matematica dell'Università di Trento \\ via Sommarive 14, 38050 Povo (Trento), Italia \\ Phone: +39-461-881635. Fax: +39-461-881624. \\ $E$-mail: visintin@science.unitn.it
}

Abstract
The concept of hysteresis operator is outlined, and some simple models are illustrated.

Some differential equations with hysteresis are also briefly discussed.

\section{Keywords}

Hysteresis, hysteresis operators, differential equations

\section{HYSTERESIS}

Hysteresis appears in several phenomena, in physics, engineering, chemistry, biology, economics, and others. Typical examples are plasticity, ferromagnetism, ferroelectricity, and so on.

A Hysteresis Loop. Let us consider the continuous hysteresis loop outlined in Figure 1.

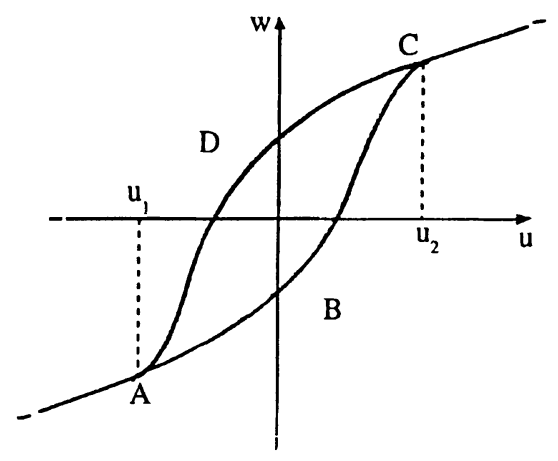

Figure 1 A continuous hysteresis loop.

If $u$ increases from $u_{1}$ to $u_{2}$, then the pair $(u, w)$ moves along the curve $A B C$; conversely, 
if $u$ decreases from $u_{2}$ to $u_{1}$, then $(u, w)$ moves along the path $C D A$. Moreover, if $u$ inverts its motion when $u_{1}<u(t)<u_{2}$, then $(u, w)$ moves into the interior of $\mathcal{L}$ (the region bounded by $A B C D A$ ) along a curve which must be prescribed by the specific hysteresis model.

At any instant $t, w(t)$ depends on the previous evolution of $u$ (memory effect), and on the initial state of the system. In the most simple setting one assumes a dependence of the form

$w(t)=[\mathcal{F}(u)](t) \quad \forall t \in[0, T]$

Here $\mathcal{F}$ represents an operator acting in an appropriate space of time dependent functions, e.g. $C^{0}([0, T])$. Obviously $\mathcal{F}$ must be causal: the output $w(t)$ may not depend on $u_{\mid l t, T]}$. The definition of $\mathcal{F}$ must include information about a desired initial state, which may then be modified because of the value of $v(0)$. For instance, an initial value $w^{0}$ such that $\left(v(0), w^{0}\right) \notin \mathcal{L}$ cannot be attained; one may then set $[\mathcal{F}(v)](0)$ equal to the projection of $w^{0}$ onto $\{(v(0), w) \in \mathcal{L}: w \in \mathbf{R}\}$.

Here it is implicitly assumed that the pair $(u(t), w(t))$ characterizes the initial state of the system at any instant $t$. However in several cases the state also depends on inner variables, whose initial value must then be specified.

Rate Independence. This means that the pair $(u(t), w(t))$ is invariant with respect to any increasing $C^{\infty}$-diffeomorphism $\varphi:[0, T] \rightarrow[0, T]$ :

$\mathcal{F}(u \circ \varphi)=\mathcal{F}(u) \circ \varphi \quad$ in $[0, T]$.

This property is the characteristic feature of hysteresis. Any rate independent and causal operator will be named a hysteresis operator.

This concept raises several problems: the formulation of examples, their adequacy to represent specific applicative phenomena, the analysis of their properties (continuity in various functional spaces, construction of the closure of the graph of discontinuous operators), their characterization, the identification of parameters, and so on.

Hysteresis is often associated with irreversibility and dissipation. As we shall see, it may have a regularizing effect.

\section{CONTINUOUS HYSTERESIS}

Here we review the main features of some simple hysteresis models.

Stop and Play. The classical Prandtl model of elasto-plasticity, also named stop, can be represented by the following variational inequality

$|w| \leq 1, \quad\left(\frac{d u}{d t}-\frac{d w}{d t}\right)(w-v) \geq 0 \quad \forall v,|v| \leq 1$

cf. Figure 2(a). For any $u \in W^{1,1}(0, T)$ and $w^{0} \in[-1,1]$, there exists one and only one $w:=\mathcal{G}(u)$ which fulfils $(3)$ and the initial condition $w(0)=w^{0}$. 
The play operator can be also represented by a variational inequality:

$|u-w| \leq 1, \quad \frac{d w}{d t}(u-w-v) \geq 0 \quad \forall v,|v| \leq 1$,

cf. Figure 2(b). For any $u \in W^{1,1}(0, T)$ and $w^{0} \in \mathbf{R}$, such that $\left|w^{0}-u(0)\right| \leq 1$, there exists one and only one $w:=\mathcal{E}(u)$ which fulfils (4) and such that $w(0)=w^{0}$.
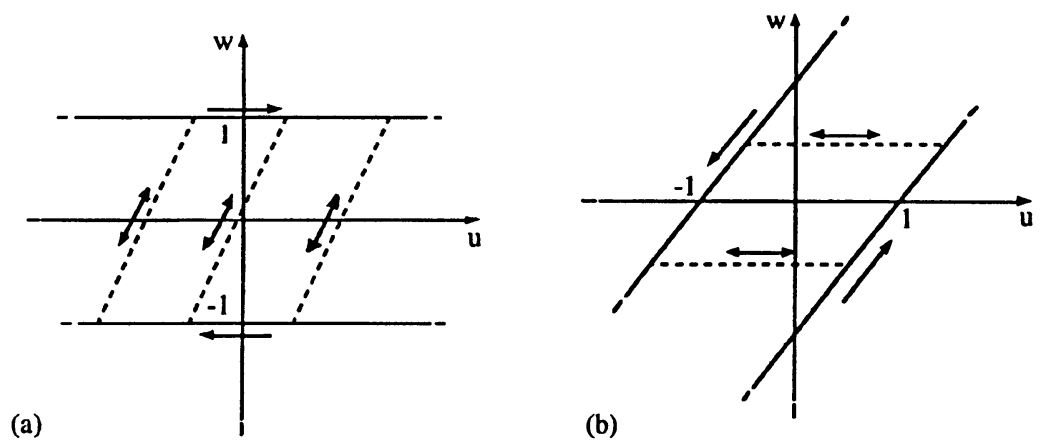

Figure 2 Prandtl's model (or stop) (a), and play (b).

Theorem $1 \mathcal{G}, \mathcal{E}$ are continuous hysteresis operator acting in $C^{0}([0, T])$. Moreover

$\mathcal{E}: C^{0}([0, T]) \rightarrow C^{0}([0, T]) \cap B V(0, T)$.

In fact $\mathcal{E}(u)$ is piecewise monotone, since the uniformly continuous function $u$ cannot have an infinite number of oscillations of amplitude larger than 1 .

Plays and stops are closely related. Denoting the identity by Id, it is easy to see that

$\mathcal{E}+\mathcal{G}=\mathrm{Id}, \quad 2(\mathrm{Id}+\mathcal{E})^{-1}=\mathrm{Id}+\mathcal{G}$.

The Duhem Model. A model of hysteresis was proposed by Duhem about a century ago. In a simplified form, it reads as follows. Let $g_{1}$ and $g_{2}$ be given nonnegative continuous functions. For any $u \in W^{1,1}(0, T)$ and $w^{0} \in \mathbf{R}$, consider the following Cauchy problem

$\left\{\begin{array}{l}\left.\frac{d w}{d t}=g_{1}(u, w)\left(\frac{d u}{d t}\right)^{+}-g_{2}(u, w)\left(\frac{d u}{d t}\right)^{-} \quad \text { in }\right] 0, T[, \\ w(0)=w^{0} .\end{array}\right.$ 
Here it is implicitly assumed that $d t>0$ : as we said, hysteresis is irreversible. Hence dividing both members by $\frac{d u}{d t}$, formally we get the equivalent condition

$$
\frac{d w}{d u}=\left\{\begin{array}{cl}
g_{1}(u, w) & \text { if } d u>0, \\
g_{2}(u, w) & \text { if } d u<0,
\end{array} \quad \text { in }\right] 0, T[.
$$

Under appropriate regularity conditions, two systems of curves in the $(u, w)$-plane are then obtained by integrating the fields $g_{1}$ and $g_{2}$. They represent the paths of evolution of the pair $(u, w)$ for increasing and decreasing $u$, respectively, and may span the whole plane $\mathbf{R}^{2}$.

A more interesting setting is obtained when $(u, w)$ is confined to a region $\mathcal{L} \subset \mathbf{R}^{2}$, bounded by the graphs of two nondecreasing continuous functions $\gamma_{\ell}, \gamma_{r}: \mathbf{R} \rightarrow[-\infty,+\infty]$ $\left(\gamma_{r} \leq \gamma_{\ell}\right)$.

Theorem 2 Assume that $g_{1}, g_{2}$ are continuous, and

$\left|g_{i}\left(u, w_{1}\right)-g_{i}\left(u, w_{2}\right)\right| \leq L(u)\left|w_{1}-w_{2}\right| \quad \forall u, w_{1}, w_{2} \in \mathbf{R}(i=1,2)$,

with $L: \mathbf{R} \rightarrow \mathbf{R}^{+}$continuous. Then for any $u \in W^{1,1}(0, T)$ and any $w^{0} \in \mathbf{R}$, there exists a unique solution $w \in W^{1,1}(0, T)$ of $(7)$, such that $w(0)=w^{0}$.

The hysteresis operator $\mathcal{D}: u \mapsto w$ is strongly continuous in $W^{1, p}(0, T)$, for any $p \in$ $[1,+\infty]$.

This operator is piecewise monotone; in general it is not continuous with respect to the weak topology of $W^{1, p}(0, T)$, for any $p \in[1,+\infty]$, and has no continuous extension to $C^{0}([0, T])$.

Note that in this model at any instant $t$ the state is characterized by the pair $(u(t), w(t))$, with no inner variable. This is not always satisfactory for applications, for ferromagnetism for instance.

Further features of these models and other examples of hysteresis operators are discussed in Visintin (1994).

\section{DISCONTINUOUS HYSTERESIS}

Relay Operator. For any pair $\rho:=\left(\rho_{1}, \rho_{2}\right) \in \mathbf{R}^{2}\left(\rho_{1}<\rho_{2}\right)$, we introduce the (delayed) relay operator

$h_{\rho}: C^{0}([0, T]) \times\{-1,1\} \rightarrow B V(0, T)$.

For any $u \in C^{0}([0, T])$ and any $\xi= \pm 1$, the function $w=h_{\rho}(u, \xi):[0, T] \rightarrow\{-1,1\}$ is defined as follows:

$w(0):= \begin{cases}-1 & \text { if } u(0) \leq \rho_{1} \\ \xi & \text { if } \rho_{1}<u(0)<\rho_{2} \\ 1 & \text { if } u(0) \geq \rho_{2}\end{cases}$ 
for any $t \in] 0, T]$, setting $\left.X_{t}:=\{\tau \in] 0, t\right]: u(\tau)=\rho_{1}$ or $\left.\rho_{2}\right\}$,

$w(t):= \begin{cases}w(0) & \text { if } X_{t}=\emptyset, \\ -1 & \text { if } X_{t} \neq \emptyset \text { and } u\left(\max X_{t}\right)=\rho_{1} \\ 1 & \text { if } X_{t} \neq \emptyset \text { and } u\left(\max X_{t}\right)=\rho_{2}\end{cases}$

Thus $w$ is uniquely defined in $[0, T]$. For instance, let $u(0)<\rho_{1}$; then $w(0)=-1$, and $w(t)=-1$ as long as $u(t)<\rho_{2}$; if at some instant $u$ reaches $\rho_{2}$, then $w$ jumps up to 1 , where it remains as long as $u(t)>\rho_{1}$; if later $u$ reaches $\rho_{1}$, then $w$ jumps down to -1 ; and so on, cf. Figure 3 .

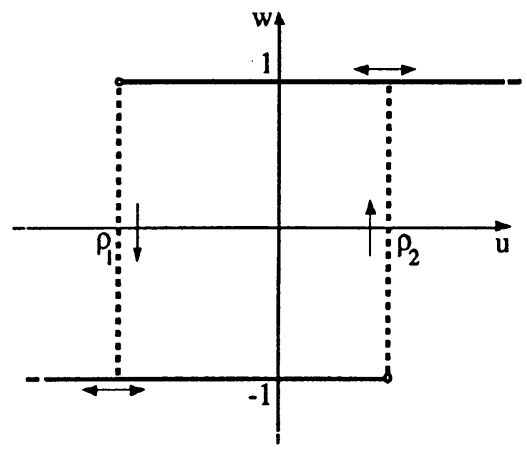

Figure 3 Relay operator.

For any function $u \in C^{0}([0, T])$, the number of oscillations of $u$ between $\rho_{1}$ and $\rho_{2}$ is necessarily finite, because of the uniform continuity; hence $w$ can just have a finite number of jumps between -1 and 1 , if any. Therefore $w$ is piecewise constant and its total variation in $[0, T]$ is finite.

It is straightforward to check that $w$ is also continuous on the right in $[0, T[$, and that $h_{\rho}$ is rate independent. Thus $h_{\rho}$ is a (discontinuous) hysteresis operator.

Remark 1 Generally speaking, hysteresis is strictly related to multistability: it can be regarded as a rule for selecting the actual value on the basis of previous evolution. In the case of the relay $w$ tends to stay constant, as long as the value of $u$ allows $w= \pm 1$.

Closure of the Relay Operator. For any $\xi \in\{-1,1\}$, the graph of the operator $h_{\rho}(\cdot, \xi)$ is not closed with respect to the strong topology of $C^{0}([0, T])$ for the input $u$, and the weak star topology of $B V(0, T)$ for the output $w$. As a counterexample it suffices to take $\xi=-1,\left\{u_{n}=\rho_{2}-\frac{1}{n}\right\}_{n \in \mathrm{N}}$ :

On the basis of the latter remark, we introduce the multivalued operator

$\bar{h}_{\rho}: C^{0}([0, T]) \times\{-1,1\} \rightarrow \mathcal{P}(B V(0, T))$ (power set) 
defined as follows. For any $u \in C^{0}([0, T])$ and any $\xi \in\{-1,1\}, w \in \bar{h}_{\rho}(u, \xi)$ if and only if $w(0) \in \begin{cases}\{-1\} & \text { if } u(0)<\rho_{1}, \\ \{-1, \xi\} & \text { if } u(0)=\rho_{1}, \\ \{\xi\} & \text { if } \rho_{1}<u(0)<\rho_{2} \\ \{\xi, 1\} & \text { if } u(0)=\rho_{2} \\ \{1\} & \text { if } u(0)>\rho_{2}\end{cases}$

and, for any $t \in[0, T]$,

$w(t) \in \begin{cases}\{-1\} & \text { if } u(t)<\rho_{1} \\ \{-1,1\} & \text { if } \rho_{1} \leq u(t) \leq \rho_{2} \\ \{1\} & \text { if } u(t)>\rho_{2}\end{cases}$

$\begin{cases}\text { if } u(t) \neq \rho_{1}, \rho_{2}, & \text { then } w \text { is constant in a neighbourhood of } t, \\ \text { if } u(t)=\rho_{1}, & \text { then } w \text { is nonincreasing in a neighbourhood of } t, \\ \text { if } u(t)=\rho_{2}, & \text { then } w \text { is nondecreasing in a neighbourhood of } t .\end{cases}$

Such a function $w$ exists, is measurable, and belongs to $B V(0, T)$.

Loosely speaking, the graph of $\bar{h}_{\rho}$ in the $(u, w)$-plane is obtained by adding the points $\left(\rho_{1}, 1\right)$ and $\left(\rho_{2},-1\right)$ to the graph of $h_{\rho}$, and then imposing the restrictions $(16)_{2},(16)_{3}$.

Theorem 3 (Closure) For any $\xi \in\{-1,1\}, \bar{h}_{\rho}(\cdot, \xi)$ is the closure of $h_{\rho}(\cdot, \xi)$ with respect to the strong topology of $C^{0}([0, T])$ for the input $u$, and the weak star topology of $B V(0, T)$ for the output $w$.

\section{HYSTERESIS, REGULARIZATION FOR O.D.E.S}

A Simple O.D.E. without Hysteresis. Ordinary and partial differential equations (O.D.E.s and P.D.E.s) can be coupled with hysteresis laws, which can be represented by means of hysteresis operators.

Let us first consider a simple O.D.E. without hysteresis:

$\frac{d y}{d t}+\operatorname{sign}_{0}(y)=f(t) \quad$ in $] 0, T[$

where $f \in L^{1}(0, T)$, and $\operatorname{sign}_{0}(\xi):=-1$ if $\xi<0, \operatorname{sign}_{0}(0):=0, \operatorname{sign}_{0}(\xi):=1$ if $\xi>0$.

This equation can represent evolution of temperature (without diffusion), in presence of a source of intensity $f$ and of a thermostat (without hysteresis), which tends to force the temperature $y=0$. Here $\operatorname{sign}_{0}(y)=-1\left(\operatorname{sign}_{0}(y)=1\right.$, respect.) means that the thermostat is providing (subtracting, respect.) heat, and $\operatorname{sign}_{0}(y)=0$ that it is switched off.

Now for a large class of functions $f$, e.g. for $f=$ Constant $\epsilon]-1,0[\cup] 0,1[$, the problem obtained coupling (17) with the initial condition $y(0)=0$ has no solution $y \in W^{1,1}(0, T)$.

This formulation can be easily modified, to allow for existence of a solution; but modifications must be justified from the modelling viewpoint. For instance one might replace 
the single-valued function sign $n_{0}$ by the maximal monotone multivalued function sign (with $\operatorname{sign}(0):=[-1,1]$, the whole interval). However values $w \notin\{-1,0,1\}$ require an interpretation.

Hysteresis Regularization. It looks more sensible to account for some hysteresis in the behavior of the thermostat, as it is often evident in practice. Let us fix any $\rho:=\left(\rho_{1}, \rho_{2}\right)$ $\left(\rho_{1}<0<\rho_{2}\right)$, define $\bar{h}_{\rho}$ as in Sect. 3 , and replace (17) by

$$
\left\{\begin{array}{l}
\frac{d y}{d t}+w=f(t) \\
w \in \bar{h}_{\rho}\left(y, w^{0}\right)
\end{array} \quad \text { in }\right] 0, T[.
$$

By the closure property stated Theorem 3, for any $f \in L^{1}(0, T)$ the Cauchy problem governed by (18) has one (and only one) solution, which also depends continuously on the data. Note that after a transient $y$ periodically oscillates between the values $\rho_{1}, \rho_{2}$, and that $d y / d t \in B V(0, T)$ if $f \in L^{1}(0, T)$.

Generalization. Let us consider an equation of the form

$$
\left.\frac{d y}{d t}+\varphi(y)=f(t) \quad \text { in }\right] 0, T[
$$

This equation has a solution whenever $\varphi$ is bounded and is of the form $\varphi=\sum_{i=0}^{4} \varphi_{i}$. Here $\varphi_{0}$ is a continuous function, $\varphi_{1}$ is a maximal monotone graph, $\varphi_{2}$ is an antimonotone function (taking two values at the point of discontinuity), $\varphi_{3}$ and $-\varphi_{4}$ are relay operators. It can also be shown that if $\varphi_{3} \equiv 0$ then there exist a maximal and a minimal solution.

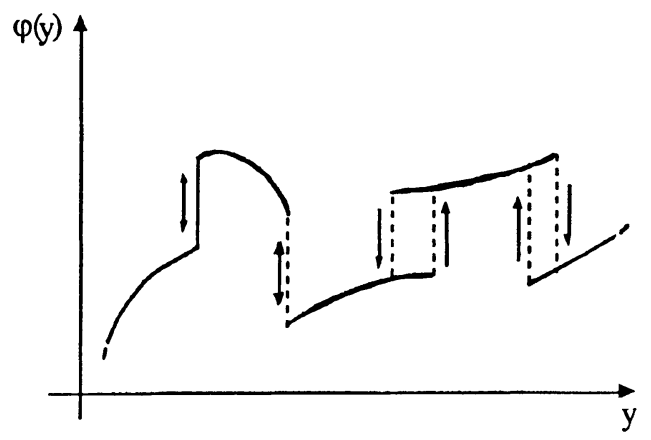

Figure 4 Example of function $\varphi$ such that equation (19) has a solution. 


\section{P.D.E.S WITH HYSTERESIS}

Two Classes of P.D.E.s with Hysteresis. Let $\Omega$ be a domain of $\mathbf{R}^{N}, A$ an elliptic operator, $\mathcal{F}$ a (continuous) hysteresis operator, and consider the equations

$$
\begin{array}{lr}
\frac{\partial}{\partial t}[u+\mathcal{F}(u)]+A u=f & \text { in } \Omega \times] 0, T[, \\
\frac{\partial}{\partial t} u+A u+\mathcal{F}(u)=f & \text { in } \Omega \times] 0, T[
\end{array}
$$

where $f$ is a given function. Of course each of these equations must be coupled with suitable limit conditions.

For $A=\operatorname{curl}^{2}$ if $N=3\left(A=-\frac{d^{2}}{d x^{2}}\right.$ if $\left.N=1\right),(20)$ can represent the evolution of a ferromagnetic system. (21) is a model of heat diffusion in presence of a distribution of thermostats.

Hysteresis and Monotonicity. A natural question concerns the classification of equation (20). If the operator $\mathcal{F}$ fulfils some monotonicity property, then (21) can be regarded as forward parabolic, and it is then natural to impose an initial condition on $u+\mathcal{F}(u)$.

The standard $L^{2}$-monotonicity is too strong a requirement for hysteresis operators. In fact it is easy to find a counterexample whenever a rate independent loop can occur.

In several cases, but not always, order preservation is fulfilled:

$$
\begin{aligned}
& \left.\left.\forall\left(u_{1}, w_{1}^{0}\right),\left(u_{2}, w_{2}^{0}\right) \in \operatorname{Dom}(\mathcal{F}), \forall t \in\right] 0, T\right], \\
& \text { if } u_{1} \leq u_{2} \text { in }[0, t] \text { and } w_{1}^{0} \leq w_{2}^{0}, \text { then } \\
& {\left[\mathcal{F}\left(u_{1}, w_{1}^{0}\right)\right](t) \leq\left[\mathcal{F}\left(u_{2}, w_{2}^{0}\right)\right](t) .}
\end{aligned}
$$

The following property of piecewise monotonicity preservation (more briefly, piecewise monotonicity) seems to be especially appropriate for hysteresis operators:

$\left\{\begin{array}{l}\forall v \in C^{0}([0, T]), \forall\left[t_{1}, t_{2}\right] \subset[0, T], \\ \text { if } v \text { is either nondecreasing or nonincreasing in }\left[t_{1}, t_{2}\right], \text { then } \\ \left\{[\mathcal{F}(v)]\left(t_{2}\right)-[\mathcal{F}(v)]\left(t_{1}\right)\right\}\left[v\left(t_{2}\right)-v\left(t_{1}\right)\right] \geq 0 .\end{array}\right.$

That is, $\frac{d w}{d t} \frac{d u}{d t} \geq 0$ a.e. in $] 0, T\left[\right.$, whenever $u, w:=\mathcal{F}(u) \in W^{1,1}(0, T)$. This means that hysteresis branches are nondecreasing.

\section{AN EXISTENCE RESULT}

Let $\Omega \subset \mathbf{R}^{N}(N \geq 1)$ be a bounded domain of Lipschitz class, $T>0$, and set $Q:=$ $\Omega \times] 0, T[$ Let

$$
\mathcal{F}: C^{0}([0, T]) \rightarrow C^{0}([0, T])
$$


be a causal and continuous operator, that is,

$\forall v_{1}, v_{2} \in C^{0}([0, T]), \forall t \in[0, T]$, if $v_{1}=v_{2}$ in $[0, t]$ then $\left[\mathcal{F}\left(v_{1}\right)\right](t)=\left[\mathcal{F}\left(v_{2}\right)\right](t)$,

$\forall\left\{v_{n} \in C^{0}([0, T])\right\}_{n \in \mathrm{N}}$, if $v_{n} \rightarrow v$ uniformly in $[0, T]$

then $\mathcal{F}\left(v_{n}\right) \rightarrow \mathcal{F}(v)$ uniformly in $[0, T]$.

Model Problem. We set $V:=H_{0}^{1}(\Omega)$, identify the space $L^{2}(\Omega)$ to its dual $L^{2}(\Omega)^{\prime}$, and the latter to a subspace of $V^{\prime}$. This yields the Hilbert triplet $V \subset L^{2}(\Omega)=L^{2}(\Omega)^{\prime} \subset V^{\prime}$, with continuous, dense and compact injections. We define the operator $A: V \rightarrow V^{\prime}$ by

$$
{ }_{V^{\prime}}\langle A u, v\rangle_{V}:=\int_{\Omega} \nabla u \cdot \nabla v d x \quad \forall u, v \in V,
$$

so that $A u=-\Delta u$ in $\mathcal{D}^{\prime}(\Omega)$. We assume that

$u^{0}, w^{0} \in L^{2}(\Omega), \quad\left(u^{0}, w^{0}\right) \in \mathcal{L}$ a.e. in $\Omega, \quad f \in L^{2}\left(0, T ; V^{\prime}\right)$.

Problem 1. To find $u: \Omega \rightarrow C^{0}([0, T])$ measurable, such that

$$
\begin{aligned}
& u \in L^{2}(0, T ; V), \quad \mathcal{F}(u) \in L^{2}(Q), \quad u+\mathcal{F}(u) \in H^{1}\left(0, T ; V^{\prime}\right), \\
& \left.\frac{\partial}{\partial t}[u+\mathcal{F}(u)]+A u=f \quad \text { in } V^{\prime}, \text { a.e. in }\right] 0, T[ \\
& {\left.[u+\mathcal{F}(u)]\right|_{t=0}=u^{0}+w^{0} \quad \text { in } V^{\prime} .}
\end{aligned}
$$

Obviously (29) is a weak formulation of (20).

Theorem 4 (Existence) Assume that (24) - (27) hold. Let $\mathcal{F}$ be piecewise monotone, cf. (23), and affinely bounded, i.e.,

$\exists L, M \in \mathbf{R}^{+}: \forall v \in C^{0}([0, T]),\|\mathcal{F}(v)\|_{C^{0}([0, T])} \leq L\|v\|_{C^{0}([0, T])}+M$.

Moreover let

$u^{0} \in V, \quad f \in L^{2}(Q) \cap W^{1,1}\left(0, T ; V^{\prime}\right)$.

Then Problem 1 has at least one solution such that

$u \in H^{1}\left(0, T ; L^{2}(\Omega)\right) \cap L^{\infty}(0, T ; V), \quad \mathcal{F}(u) \in L^{2}\left(\Omega ; C^{0}([0, T])\right)$.

For the proof we refer to Visintin (1994; Chap. IX).

For a large class of hysteresis operators (including plays, for instance) the previous assumptions are fulfilled, and continuous and monotone dependence on the data (whence uniqueness of the solution) can be proved.

In case of discontinuous hysteresis operators some modifications of the setting of Problem 1 are needed. In that case however well-posedness can also be proved. 


\section{REFERENCES}

Here are some basic references to the mathematics of hysteresis.

Bossavit, A., C. Emson, C. and Mayergoyz, I.D. (1991) Géométrie différentielle, éléments finis, modèles d' hystérésis. Eyrolles, Paris.

Brokate, M. (1987) Optimale Steuerung von gewöhnlichen Differentialgleichungen mit Nichtlinearitäten vom Hysteresis-Typ. Lang, Frankfurt am Main. English translation: Optimal control of ordinary differential equations with nonlinearities of hysteresis type. In: Automation and Remote Control, 52 (1991), 53.

Brokate, M., et al. Contributions to the session on "Problems in hysteresis". In: Proceedings of World Congress of Nonlinear Analysts, Tampa, August 1992 (to appear).

Brokate, M., et al. (Visintin, A. ed.) (1994) Phase Transitions and Hysteresis. Lecture Notes in Mathematics, vol. 1584. Springer, Berlin.

Brokate, M. and Sprekels, J. Hysteresis phenomena in phase transitions (Monograph in preparation).

Krasnosel'skiǐ, M.A. and Pokrovskiı̌, A.V. (1989) Systems with hysteresis. Springer, Berlin (Russian edition: Nauka, Moscow 1983).

Krejčí, P. Convexity, hysteresis and dissipation in hyperbolic equations. (Monograph in preparation).

Mayergoyz, I.D. (1991) Mathematical models of hysteresis. Springer, New York.

Visintin, A. (ed.) (1993) Models of hysteresis. Longman, Harlow.

Visintin, A. (1994) Differential models of hysteresis. Springer, Berlin. 